\title{
Flexible Content Browsing for Impaired e-Learners
}

\author{
B.L.Velammal \\ Assistant Professor, \\ Department of Computer Science, \\ Anna University, Chennai, India
}

\author{
P.Anandha Kumar \\ Associate Professor, \\ Department of Information Technology, \\ MIT Campus, Anna University, Chennai, India
}

\begin{abstract}
Today web sites are designed in graphical mode for interaction with insufficient user assistance. Keen-sighted users can identify the content and quickly recognize relevant information in Web pages. On the contrary, individuals with visual disabilities have to use screen-readers to browse the Web. In this paper, we address the problem of information display in a non-visual Web interface. Access is made using the notion of context by assisting through the audio support and embodying our approach, by providing the standard features of a screen-reader along with browsing through voice browser. However, when a user follows a link, it captures the context of the link using a simple topic-boundary detection technique, and uses it to identify relevant information on the next page with the help of screen readers, and navigation is done through the voice browser. In order to aid the visually impaired users with a complete user friendly browsing approach we permit the content to be known by the screen-readers and voice browsers for the purpose of navigation.
\end{abstract}

\section{General Terms}

Content adaptation, Human computer interaction.

\section{Keywords}

e-content adaptation, voice content, content adaptation, e-learning, impaired assistance.

\section{INTRODUCTION}

Web has become an indispensable source of information and we use it inevitably in our daily lives. The primary mode of interaction with the web is via a well designed graphical user interface. Browsers are designed to aid users with visual interaction that support distinctive content and layout. As we browse the Web, it is important to find the relevant content at the right site on the webpage. Manual browsing of web pages doesn't permit the content to be browsed easily, as it is difficult to predict the content. In such a situation, it is important to assist the user with supportive devices.

A screen reader is a software application that attempts to identify and interpret what is being displayed on the screen. A voice browser presents information aurally, using pre-recorded audio file playback or using text-to-speech software to render textual information as audio. These aids the users for navigation purpose also, say for example, navigation from the current page to the previous page can be performed by aurally rendering the command "back". Keen-sighted individuals can process visual data and quickly locate relevant information. Often, this task can be time-consuming and strenuous for visually impaired individuals. Typically, visually impaired people browse the web using screen readers. Many screen-readers process web pages sequentially, i.e. they read through the menus, banners, commercials or anything else that comes before the desired information, thereby increasing the browsing time. To alleviate this problem, screen-readers provide shortcuts to skip segments of text in the order they appear on the page. Nevertheless, users may still have to listen or skip through substantial page content before they get to the desired information. To help users locate the information quicker, most screen- readers allow keyword searching. This assumes that the users know what they are looking for. However, simple searching has two problems: it works only for exact string matching and it declines users in case of a wrong match. In both cases, users have to start searching from the beginning of the page to locate the relevant content quickly and efficiently. This in turn, makes browsing easy and simple for visually disabled people.

\section{RELATED WORK}

In the last few years, while considering the development of the screen reader with early operating systems, such as MS-DOS, which employed command line interfaces, the screen display consisted of characters mapping directly to a screen buffer in memory and a cursor position [6]. Input was through the keyboard. All this information could therefore be obtained from the system, either by hooking the flow of information around the system and reading the screen buffer or by using a standard hardware output socket and communicating the results to the user.

Later, the Research Centre for the Education of the Visually Handicapped at the University of Birmingham developed Screen Reader for the Micro and Portable devices, so that they can navigate and adapt to the environment of the blind users. With the arrival of graphical user interfaces (GUIs), the situation became more complicated. A GUI has characters and graphics drawn on the screen at particular positions, and as such there is no purely textual representation of the graphical contents of the display. Screen readers were therefore forced to employ new low-level techniques, gathering messages from the operating system and using these to build up an "off-screen model", a representation of the display [7] in which the required text content is stored. For example, the operating system might send messages to draw a command button and its caption. These messages are intercepted and used to construct the off-screen model. The user can switch between controls (such as buttons) available on the screen and the captions and control contents will be read aloud and/or shown on refreshable Braille display.

Screen readers can also communicate information on menus, controls, and other visual constructs to permit blind users to 
interact with these constructs. However, maintaining an off-screen model is a significant technical challenge: hooking the low-level messages and maintaining an accurate model are both difficult tasks.

A relatively new development in the field is web-based applications like spoken-web [2] that is a web portal, managing content like news updates, weather, science and business articles for visually impaired or blind computer users, or browse aloud that adds text-to-speech functionality to web content. The primary audience for such applications is those who have difficulty in reading because of learning disabilities or language barriers. Although functionality remains limited compared to equivalent desktop applications, the major benefit is to increase the accessibility of verbal websites when viewed on public machines where users do not have permission to install custom software, giving people greater freedom to roam.

Apart from desktop usage, wide spread of portable devices like mobiles are used for accessing information from anywhere in the world. Context aware formats [3] have been introduced to reduce the searching of content and limit to the required output. But this kind of mobile usage is mainly focused for knowledge industry and not for conventional use. Explicit cache replacement [5] policies have also been studied for better resource allocation and obtaining a relatively better response, where in the output depends the size of the content that is being accessed and simultaneous requests from different users.

\section{CONTENT ADAPTATION}

\subsection{Assist the disabled user for e-learning}

The evolution of an Information Society has transformed many activities in our everyday lives for disable users too, including how we work, communicate, entertain, teach and learn. More specifically, in recent years widespread Internet connectivity, together with the development of new web-based multimedia technologies, has strongly encouraged educational uses of Information and Communication Technology. All activities that need network technologies to deliver learning and training programs can be considered forms of e-learning. Information and Communication Technology naturally fuelled the spread of e-learning for disable, forcing the emergence of E-learning that currently represents the most widespread form of Distance Education, which generally refers to educational activities that involve teachers and students remotely located both in time and space. Current distance education is based on a wide range of delivery methods, including traditional correspondence, as well as books, audio/video tapes, interactive TV, CD-ROM and DVD, as well as services that can be offered through the Internet. More generally, "e-learning" can be defined as the delivery of education or training programs through electronic means. From a technological point of view, today's e-learning is rooted primarily in a web-based delivery of educational multimedia content [4], coupled with synchronous and asynchronous communication features that allows disabled students and teachers to interact. In addition, new e-learning forms are emerging, increasing nomadic and ubiquitous access, such as narrowcasting based ones.

\subsection{Assist the disabled for content navigation}

Accessibility is generally dependent on assistive technologies used by people with disabilities to access their PCs, but it also depends on whether people with various disabilities can perform specific tasks on their PCs with the help of these assistive technologies. In this Subsection, we introduce how people with disabilities access the Web, by considering a few examples of specific disabilities and the assistive technologies that might be used. The examples are not an exhaustive list, but are intended to offer a short overview of some of the more relevant cases, where the type of disability has a significant affect on a person's ability to access the Web.

First we consider people who are blind, who will most likely use a screen reader to access their computers. A screen reader gathers information from a computer screen and outputs that information as synthesized speech. While accessing the Web, a screen reader may encounter a variety of barriers, such as uncommented images origin formation whose meaning depends on color or its position on the screen. Visual information without text alternatives that can be read by a screen reader will otherwise not be accessible to a screen reader user. Similarly, people who have poor vision may use an assistive technology that enhances their residual sight, such as a screen magnifier. This tool enlarges the information displayed on the screen and helps the user by appropriately modifying some of its characteristics such as font size, contrast or colors. The resulting display from a screen magnification tool represents only a portion of the whole screen, which often creates a loss of context.

To accommodate those using a screen magnifier, authors need to create content that can be easily resized. In addition, it is useful using relative measures instead of absolute measures to define the size characteristics of their content, allowing it to resize, to fit any size browser window without losing or distorting the information being presented. Mobility related difficulties range from simply being unable to grasp or handle a mouse, to disabilities that require the use of voice input to control a computer instead of the conventional keyboard and mouse. Generally people with mobility impairments need Web pages that can be fully accessed by using a keyboard or mouse-equivalent input device. A head mouse and single click switches might be used in place of a traditional mouse, controlling the cursor with head movements and clicking on the mouse by leaning on a large button like switch.

Alternative mouse input devices might be used together with an onscreen keyboard or voice recognition system for navigating and entering content. Finally, we can consider the less obvious or hidden disabilities such as learning disabilities and dyslexia, disabilities that affect a user's ability to read, write, navigate, comprehend and recall relevant information. People who have such disabilities may use a text-to-speech system that reads text on the screen aloud using synthesized speech. In addition, multimedia is widely used as an accessibility solution for anyone who has difficulty reading and/or understanding information presented in text form. Consistency in presentation [1] is often an important aspect for improving accessibility and usability for those with learning disabilities, such as navigation tools that remain the same throughout a Web site, a consistent look-and-feel, and page lay outs that do not change from screen to screen.

\subsection{Assist the blind user with audio support}

To assist the blind people to browse through the internet we have 
to convert the voice input to the device known URL text pattern, which requires the conversion from voice to the text and mapping of the trained dictionary words related to the URL pattern, and display in the address bar. The adaptation of content is for everyone, so to assist the blind people to browse through the internet. The functionality of the voice browser in the block diagram representation is given Fig 1 .

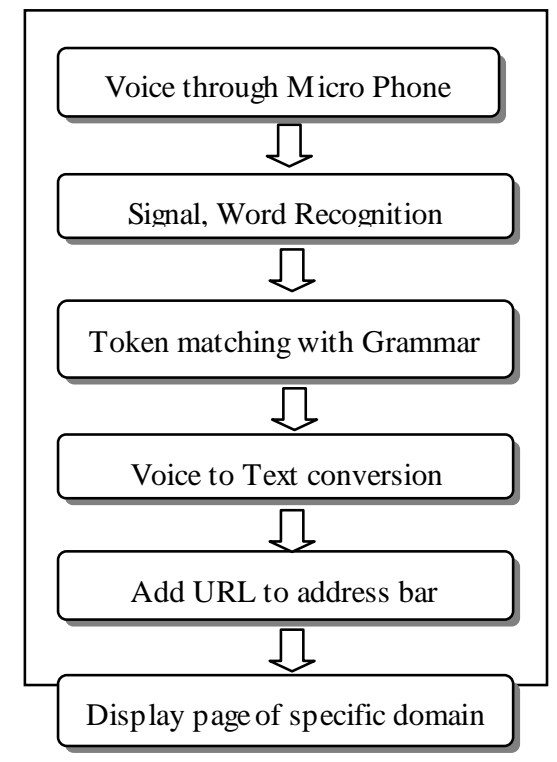

Fig 1. Block Diagram of Voice Browser

The adaptation of content is for everyone, so the content in the website should be known to the blind people. Audio from the blind people is the source of input and the text in the website are converted again as audio is the output

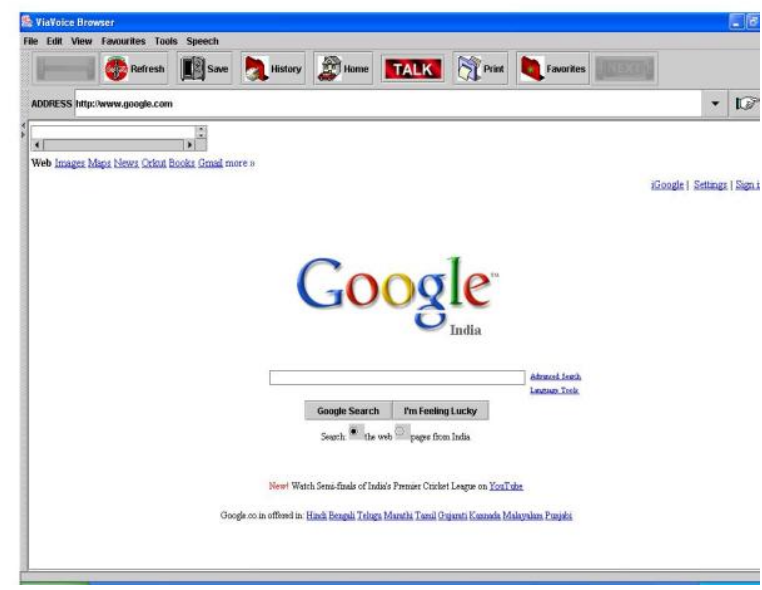

Fig 2. Web page accessed using Voice

The blind people are assisted with audio support and this involves receiving the voice through microphone, word recognition, token matching with signal, voice to text conversion and displaying the specific navigation for disabled users. Here the microphone is the hardware device used to get the audio input, finding out the word from the word recognition, matching of the voice word to the dictionary word through the token matching and finally specific navigation is done through the voice to text conversion processed further to reach user friendly browsing to the disabled users.
Browser with the navigation to save a web page is given in Fig 3.

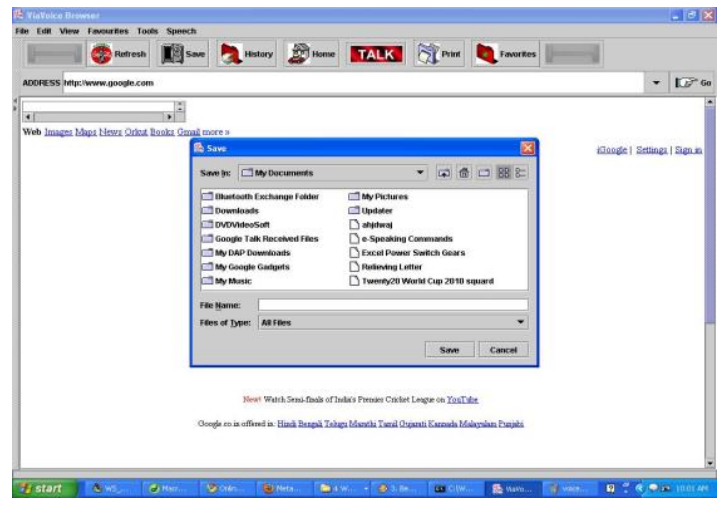

Fig 3. Voice control for saving web pages

\section{CONCLUSION \& FUTURE WORK}

In this paper, we have designed an assistive voice browser with enhanced content and context for the disabled web users, to assist the physically challenged e-learners, we consider the content to be known to the user by using screen readers and magnifiers and finally support the navigation through the voice browser which can identify the voice of the user, token match with dictionary words and finally support the menu navigation. For the fast and easy navigation we use the effective search for particular content and start screen reader to come across the e-learning environment. While comparing to the existing model, this approach provides an effective content browsing and easy accessibility of the web for the disabled users. Our results show that use of context can potentially save browsing time and substantially improve browsing experience for visually disabled people.

In the future work of this project, the assisted environment for the disabled e-learning users should be adapted to the physically disabled user's environment along with the hardware capability of the system. In order to implement the further enhancement we have to minimize the keyboard access keys and develop shortcut keys for the user to access the web easily, find the hardware capability of the system and process through the further stages according to ability of the system.

\section{REFERENCES}

[1] J. Clark. Building Accessible Websites. New Riders Publishing, Indianapolis, 2002. [1]A.Jiebo Luo, Alan Hanjalic, Qi Tian, Alejandro Jaimes (2009),'Integration of Context and Content for Multimedia Management: An Introduction to the Special Issue', Vol.11 No.2, pp.193-195.

[2] A. Celentano and O. Gaggi. Context-Aware Design of Adaptable multimodal documents. In Multimedia Tools and Applications, Vol. 29, No. 1, pages 7-28, Kluwer, April 2006.

[3] F. Cardinali. Towards Narrowcasting \& Ambient Learning:New Mobile, Location and Context Aware Formats for the Content Industry towards the Knowledge Society. In Proceedings of the E-Learning Conference, May 2005.

[4] S. C. Cheng, C. W. Su and Y. T. Lin. Mobile Learning with Intelligent Download Suggestions. In Learning Technology, Vol. 7, No. 2, IEEE Computer Society Technical Committee 
Volume 27-No.3, August 2011

on Learning Technology (LTTC), April 2005.

[5] C. Y. Chang and M. S. Chen. On exploring aggregate effect for efficient cache replacement in transcoding proxies. In IEEE Transactions on Parallel and Distributed Systems, Vol.14, No. 6, pages 611-624, IEEE Communications Society, June 2003.

[6] S. Chandra, A. Gehani, C. S. Ellis and A. Vahdat. Transcoding
Characteristics of Web Images. In Proceedings of the SPIE Conference on Multi-Media Computing and Networking (MMCN '01), Vol. 4312, pages 135-149, January 2001.

[7] S. Chandra, C. S. Ellis and A. Vahdat. Differentiated Multimedia Web Services Using Quality Aware Transcoding. In Proceedings of the 19th Annual Joint Conference of the IEEE Computer and Communications Societies (INFOCOM '00), IEEE Communications Society, March 2000. 